\title{
The Design and Realization of Digital Exhibition of Yangjiabu New Year Pictures
}

\author{
Yan Jiang \\ Computer information center \\ Beijing Fashion Technology College \\ Beijing,China \\ jsjjy@bift.edu.cn
}

\author{
Manchen Han \\ Software institute \\ Beijing University of Technology \\ Beijing,China \\ manchen@yahoo.com.cn
}

\begin{abstract}
With the development of virtual reality technology and internet shock, in order to be inherited, Yangjiabu New year pictures, as a traditional culture, need to be preserved, protected, developed and innovated with times. This paper realized the digital exhibition of Yangjiabu New Year pictures which integrate the functions of virtual roam, watching, and education using virtual reality technology. To begin with, the real world with all kinds of information related of Yangjiabu be collecting and collating; Secondly, with 3D modeling software, to complete a series of three-dimensional models; Finally, uses virtual engine UDK to achieve the virtual tour and various dynamic interaction, such as picking, rotation, particle features and door switch and so on. Besides, the development of traditional New Year pictures was analyzed. The best connection of technology and art is further explored to inherit and preserve Chinese traditional culture. This paper realized and designed demonstration system in view of interaction and roam by using 2D mapping, 3D modeling and VR technology.
\end{abstract}

Keywords- Yangjiabu New Year picture; VR technology; UDK; interaction technology; traditional culture

\section{INTRODUCTION}

New Year pictures of Yangjiabu, as an active cultural heritage, show robust vitality and great historical culture significance, and present various forms with times. It has undergone the stages of generation, development, prosperity, and declination. Public aesthetic changed with the development of agricultural economy, which was dominated by natural economy, and the integration of cultures of different nationalities and countries. This accelerated the inheritance and accumulation of Yangjiabu New Year pictures among different generations and formed its specific styles. However, with the frequent political, economical, and cultural exchanges among different countries, as well as the change of public thought, the technique of traditional New Year pictures now is likely to be lost. In recent years, the development of multimedia technology provides Chinese art field with a new platform with great vitality.

At present, China has made great effort on digital exhibition. The effort includes different technology application. For example, multimedia and digital technologies are applied as exhibition technologies; Latest science technology is combined with unique digital creativity to disseminate content; visitors are attracted by varies novelty technologies; man-machine interaction display is realized. Virtual exhibition is a beneficially supplement to physical display. Digital exhibitions are able to develop continually based on traditional exhibition, exactly as the development of high technology expands development space of exhibitions [1-4].

For example, the opening ceremony of Olympic Games in Beijing in 2008. With the development of modern science technology, the history of artificial turning background needs to be changed. Large electronic screen background display is compared with simple show. It is used to not only for saving time, labor and cost, but also presenting magical change, colorful artistic effect and modernity.

\section{THE PURPOSE AND SIGNIFICANCE OF DIGITAL}

\section{TRANSFORMATION OF YANGJIABU NEW YEAR PICTURES}

Information technology and printing have been developing with times,. New Year pictures are facing survival crisis, because the original traditional handcraft has been replaced by printing technology. Ignoring the traditional handcraft suggests losing the future[5].

This is why the Artist Feng Jicai called on rescuing the Yangjiabu pictures, and received great supports of the government. Yangjiabu New Year pictures present the history of Chinese culture development, and are human culture symbol integrated early nature worship with psychological needs[6].In the process of transformation, the core purpose is to completely inherit and spread the culture and art, and it also can be a part of archaeology. Folk culture acts as cultural exchanges among different nationalities and countries. For example, the domestic animation film ,Making Havoc in Heaven Fig .1. Images illustrate adornment style of New Year pictures using western musical instruments. Chinese traditional artistic style is presented to public with new 3D multimedia technology [7-10]. 


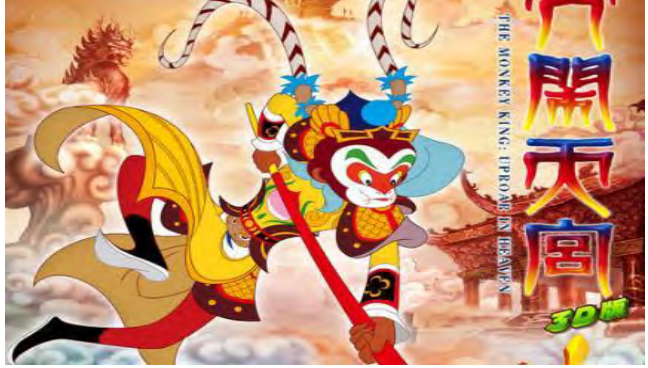

Figure 1. Making Havoc in Heaven

With the continuous improvement of multimedia technology, multimedia technology and game engine UDK technology are integrated to construct a dynamic application environment by UDK technology. The aim is to spread and inherit traditional culture efficiently. Especially, the young people nowadays pay increasing less attention on folk culture; more vitality needs to be put for the protection of the losing artistic culture. This paper focuses on the presentation of layering pictures; and interaction roam leads to more stereo and clearer display, as shown in Fig .2 and Fig .3. The reality is combined to with thought to create a virtual space and restore the workshops of Yangjiabu [11]. The 3D roaming form brings us to the world of folk culture based on the prototype Pailou and architectural appearance.

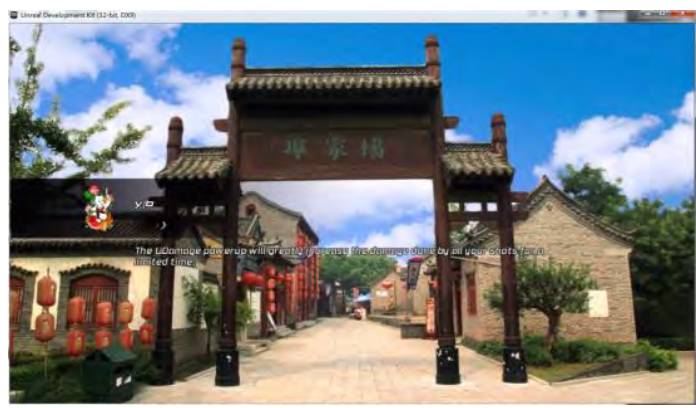

Figure 2. System Starup Interface

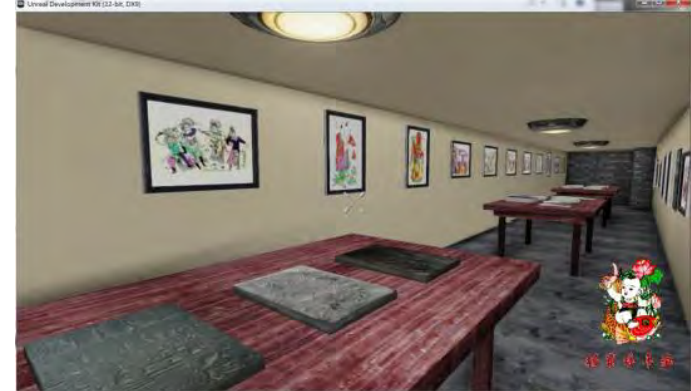

Figure 3. The Main Of Scene

\section{STRUCTURE AND CONTENT DESIGN OF THE SYSTEM}

\section{A. Digital exhibition design}

The design of virtual exhibition of Yangjiabu is formulated systematically. In the scheme, space express, function and interface are designed respectively; the scheme design and process application are briefly introduced. And all design principles accord with the characteristics of virtual technology, and lay a foundation for realizing roaming and interaction.

1) Introduction of technology scheme design and application process diagram

Software Unreal Development (UDK ) completes the pre-set roam and interaction using Photoshop, 3Dmax and UDK. Roaming and interaction technologies are introduced simply, and all designs need to be completed according to the characteristics of virtual reality technologies, such as Mapping editing, light parameter and interactive program code and so on. The detailed technology scheme is produced to satisfy analysis needs. As shown in Fig . 4

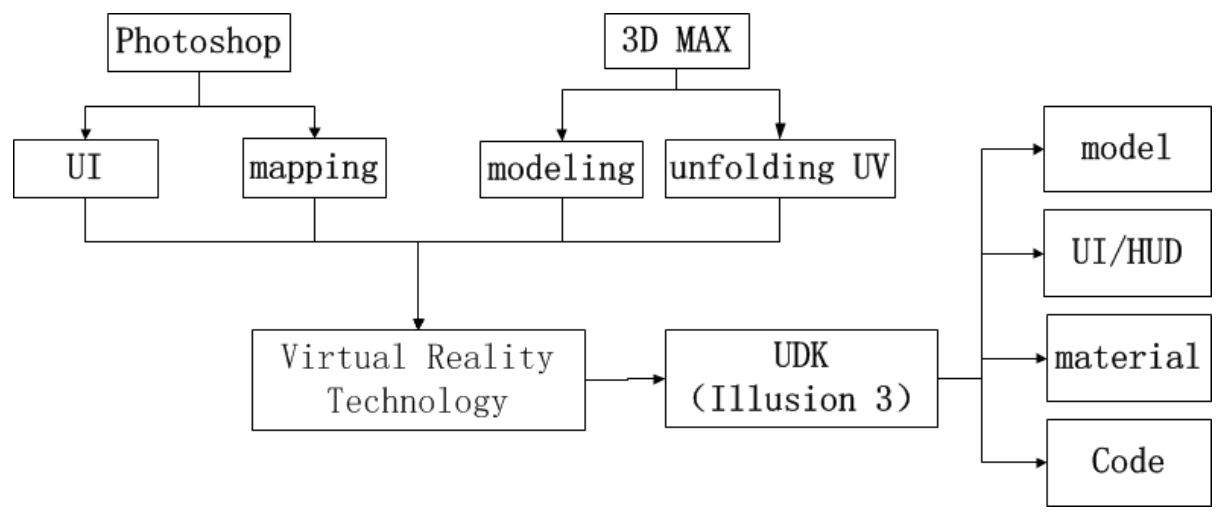

Figure 4. Technology scheme design

Meanwhile, the corresponding technology application process diagram is produced, as shown in the Fig .5.

2) Style design

In design, art scenes should be realistic as much as possible,
From an interface to a scene, each object, production and model are designed with unified style. And folk characteristics need to be illustrated in way of model, color and interaction. So user tends to be brought into the scene. Manual roaming and interaction are used to improve the mystery of production. The Layout intention of design and 
integrity of traditional folk New Year pictures workshop are used to understand the details of scenes and master the whole situation.

During the whole virtual roaming, the relation among partial model, product, interaction design and entirety are characterized by comprehensive thinking. In modeling, scene designs during the whole roaming require to be grasped in macroscopic view. Especially when objects penetrate with each other, the relations between entirety and parts are dealt to achieve coordination and unity. After conducting 3D modeling, in the importing process of UDK software, more attentions should be paid on the connected relation between scenes to ensure the continuity when the scenes are switched. To attain the full composition with free space at the same time, the main UI interface is drew using Photoshop, as shown in the Fig .6

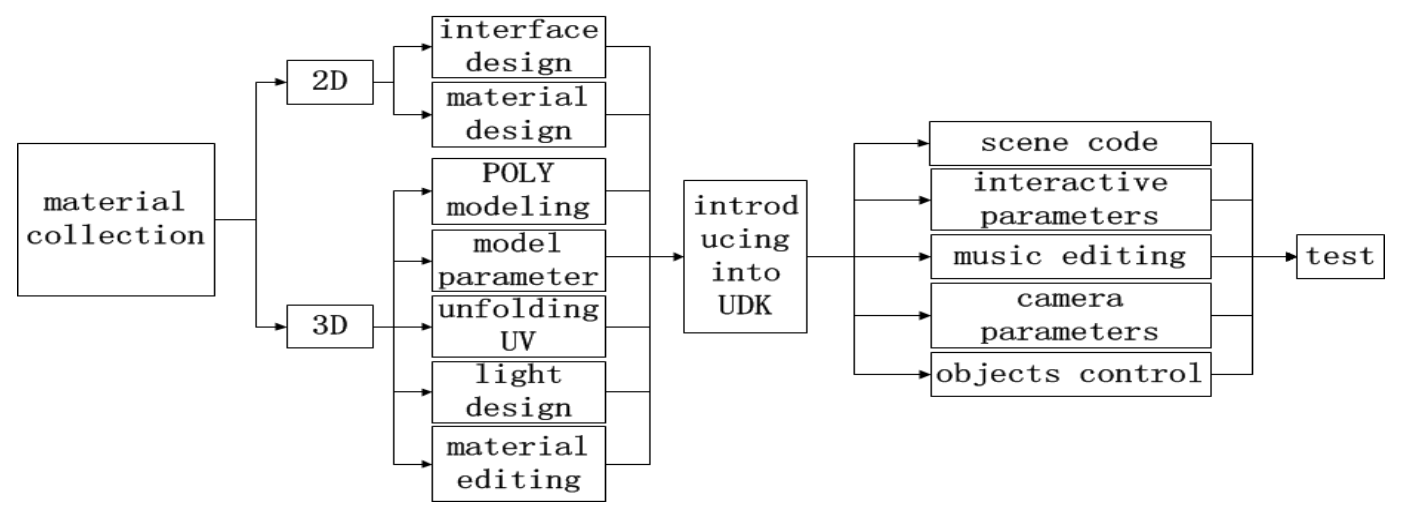

Figure 5. The process diagram of technology application

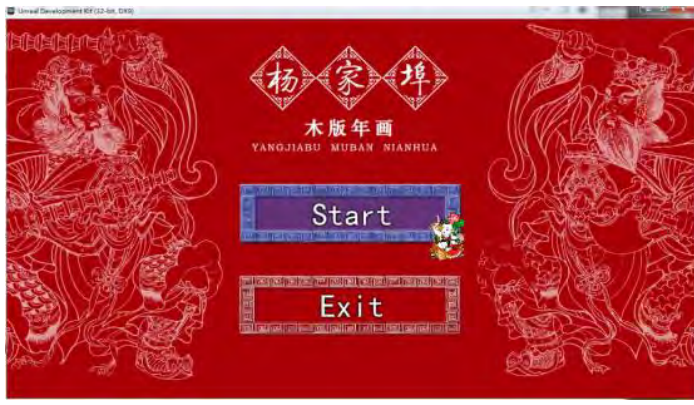

Figure 6. The main interface UI

Firstly there is suspense: users are suddenly enlightened when they come to the scene. The main interface of UI design is divided into four areas: interactive control area, the mouse following area, background keeper and button. They are designed to show folk style, simplicity and image are the basic elements of interface design. The construction has a deterministic effect on expanding scene space and roam interaction by using keyboard and mouse to adjust in the first perspective.

\section{B. Creating physical models}

The theme of paper is determined to produce a Yangjiabu exhibition by using 3D. The first step is to make a systemic project. In 3Dmax modeling, mapping and UV are used based on the original Yangjiabu construction and workshop. 3D view need to be applied skillfully, additional operation is performed in each diagram. Space arrangement is checked from perspective drawing at any time as roaming path is simulated, as shown in Fig. 7.

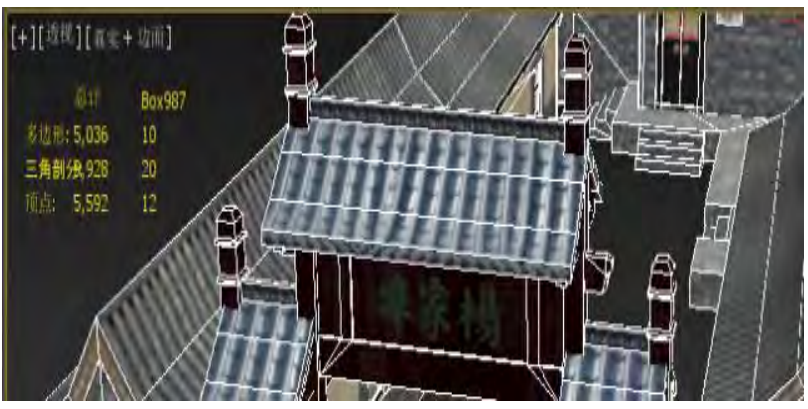

Figure 7. Perspective drawing

Here, a collision needs to be set in a model to improve system stability of dealing the collision. The collision refers to that two particles or objects are close with each other through interaction in shorter time, so their movement state changes obviously[12].

The Volume is designed to cover the model closely based on the original model. The collision occurs on the objects with fewer sides. So there will be less possibility for the objects penetrating with each other during roaming process

\section{INTERACTION FUNCTIONS OF THE SYSTEM}

The study is realized on the platform of UDK which was released by Epic co. ltd., as visual 3D and designed aiming at the next generation of gaming hardware platform. It is game engine with powerful realistic drawing effect. The engine uses NVIDIA GF6800 graphics processor which is in agreement with new standards Shader 3.0 or Microsoft Xbox2 with the function of next generation game video host. The aim is to reach high precision processing effect better than 3D diagram .

In conclusion, virtual construction, roam, realtime 3D workshop and interactive model are able to be completed with virtual reality technology. 
In the design scene, roam and interaction are demonstrated. The interaction refers to feedback among things. During human communication, the action, willingness and information are replied by the other side. This course constitutes the interaction .

1) Particles realization

Parameters are set to ensure ejecting particles in the right time. The area around the god of wealth model is choose and set "Start", the ejecting particles are presented when the scene roams in front of the god of wealth, while it stops out of the scope. Parameters are shown in the Fig .8.

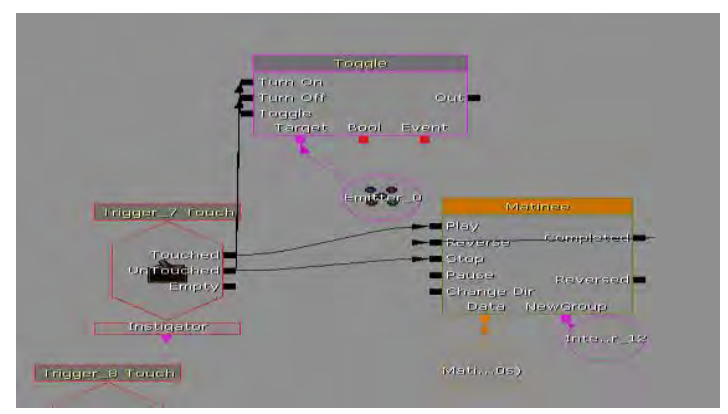

Figure 8. Start setting

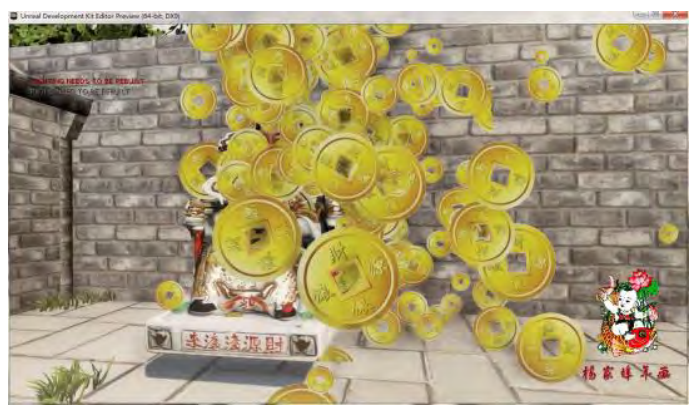

Figure 9. Particle

Meanwhile, to enhance the interest of scene interaction, the initial size and shape of particles and the lifetime need to be adjusted, as shown in Fig .9 .

2) Realization of pickup interaction

In the design, part books and pear block both have achieved pickup action. At setting time of parameters, the factors of objects ${ }^{\text {ee }}$ size and collision code need to be considered firstly, as shown in Fig .10.

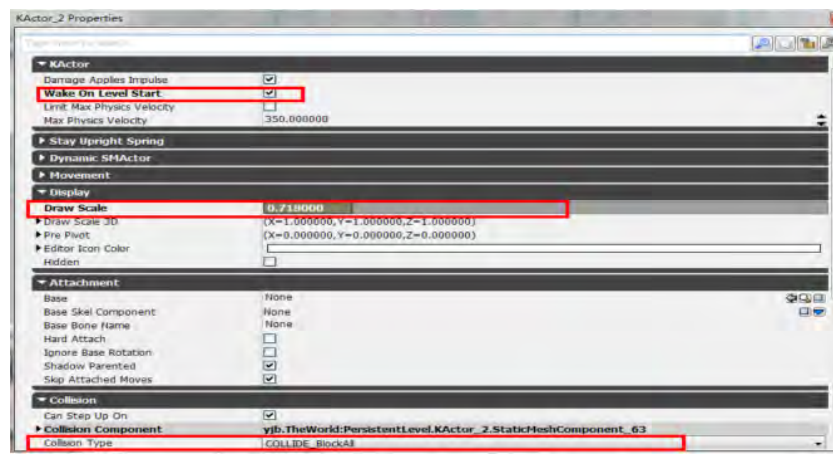

Figure 10. Collision code

3) Switch settings

To have door endowing with switching functions, the association of door and matinee is required. "add movement track" is added in"Group" in matinee, to record key frame .Time is set to the place after the key frame. After clicking "enter", then door"s setting position is pulled in the scene. Because the relative parameters are not set, "Start" can be done only once. In order to realize "Start" repeatedly, ,max count $1^{\text {ee }}$ in the attribute needs to be changed to ,, $0^{\circ e}$. Actually, Kismet was used in virtual interaction in this study, i.e. simple actions: users open a door, Kismet can be applied anywhere in which right thing is started in a key position with a certain object in the game

In conclusion, the design is to satisfy users telepresencenm according to the changes of user's location and direction and produce 3D scenes in real time. One can walk freely in the scenes. The interactive technologies include as follows, taking a book at anytime, pickup block, particle adding, dynamic switching, character model rotation and video image etc.. They are applied to produce dynamic visual effect and improve the performance function of digital exhibition of Yangjiabu New Year picture.

\section{THE SYSTEM TEST}

This system would be tested in two different models and parameters of personal computers, and the specific hardware environment are shown in table 1 . The file named "UDKInstall-yjb-nianhua.exe" would be installed on the test machine, after the success of the installation, click" finish", as shown in Fig .11.

TABLE I. The TEST OF HARDWARE ENVIRONMENT

\begin{tabular}{|c|c|c|}
\hline Type & Desktop & Notebook \\
\hline CPU & $3.40 \mathrm{GHz}$ & $2.40 \mathrm{GHz}$ \\
\hline RAM & $2 \mathrm{~GB}$ & $2 \mathrm{~GB}$ \\
\hline OS & Windows XP & Windows7 \\
\hline \multirow[t]{5}{*}{ Operation } & $\begin{array}{l}\text { Enter the software } \\
\text { rapidly, roaming and } \\
\text { interactive operation } \\
\text { smoothly }\end{array}$ & $\begin{array}{l}\text { Enter the software slower, } \\
\text { roaming and interactive } \\
\text { operation smoothly }\end{array}$ \\
\hline & 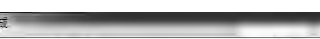 & $-\frac{x}{x}$ \\
\hline & UNREAL & \\
\hline & \multicolumn{2}{|c|}{ 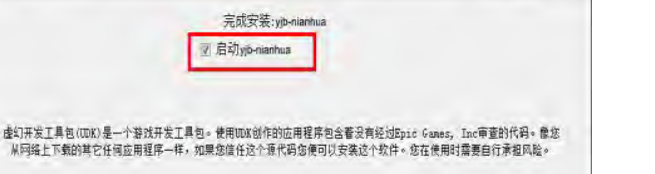 } \\
\hline & & 完成 \\
\hline
\end{tabular}

Figure 11. Complete The Installation

\section{CONCLUSION}

To meet the design requirements of VR system, this research realized the design integrating roaming and interaction using 3D and virtual reality technology based on the original appearance of Yangjiabu New Year picture exhibition. The design has been exploring. It integrates the advantages of digital technology and traditional pictures. VR technology application is favorable to inheritance and development of traditional culture. 


\section{ACKNOWLEDGMENT}

The work was supported by the High-level talents introduction and training program of Beijing colleges and universities.

\section{REFERENCES}

[1] Gengzhihong. Display Design at Digital Age[M]. Beijing: China Water \& Power Press, 2009.

[2] Mitsumasa Koyanagi,Kang-Wook Lee,Takafumi Fukushima, et al. Heterogeneous 3D Integration Technology and New 3D LSIs[C]// 2012 IEEE 11th International Conference on Solid-State and Integrated Circuit Technology: Xi'an Jiaotong University Press,2012:423-430.

[3] Zhang Yi.Virtual Reality Technology of the Modern Fashion Design[C]/2011 3rd IEEE International Conference on Information Management and Engineering: Zhengzhou University Press,2011:309-315.

[4] Janice Sorkow.Pricing and Licensing for Museum Digital Content[J]. Archives and Museum Informatics, 2004, 11(2):90107.
[5] Rendianshun. The dilemma and path of Chinese publishing digital transformation[J]. Digital Publishing, 2008,3(2):55-61.

[6] Wanglidao. The Chinese Traditional Moral Image[M]. Beijing: People's Fine Arts Publishing House, 2008.

[7] Xuzhendong. The Change of Design context Viewed from the Image Sunwukong[J], Journal of Zhejiang University of Science and Technology, 2013,3(4):142-150.

[8] Glăveanu Vlad Petre. Creating creativity: reflections from fieldwork[J]. Integrative psychological \& behavioral science, 2010, 4 (1):100-115.

[9] Sang Hyun Kim,Jeong Yeop Kim,Se Jung Kim. A Study of 3D Stereoscopic Map-Tool Using Ogre3D Game Engine[C]//2012 International Conference on Information and Computer Applications : Hong Kong Polytechnic University Press, 2012:432440.

[10] Maryam charrakh, Azita sherej sharifi.Brand Performance Based on Brand Features[C]//2011 International Conference on Innovation, Management and Service:Singapore hopes Press, 2011:123-130.

[11] Lvpintian. Chinese Folk Art Idea[M]. Changsha: Hunan Fine Arts Publishing House, 2007.

[12] Panchangxue. From the Art of Shadow Play to Interactive Media Design[J]. Decoration, 2003.11 (2): 34-41. 